\title{
An Investigation of Parameter Optimization in Fingerling Counting Problems
}

\author{
Adair da Silva Oliveira Junior ${ }^{1}$, Marcio Carneiro Brito Pache ${ }^{2,5}$, Fábio Prestes Cesar Rezende ${ }^{2}$, \\ Diego André Sant'Ana ${ }^{2,5}$, Vanessa Aparecida de Moares Weber ${ }^{2,3}$, Gilberto Astolfi, ${ }^{1,4}$, \\ Fabricio de Lima Weber ${ }^{3}$, Geazy Vilharva Menezes ${ }^{1}$, Gabriel Kirsten Menezes ${ }^{1}$, \\ Pedro Lucas França Albuquerque ${ }^{7}$, Celso Soares Costa ${ }^{2,6}$, Vanir Garcia ${ }^{2,4}$, \\ Eduardo Quirino Arguelho de Queiroz ${ }^{2}$, João Victor Araújo Rozales ${ }^{2}$, Milena Wolff Ferreira ${ }^{2}$, \\ Marco Hiroshi $\mathrm{Naka}^{2,4}$, and Hemerson Pistori ${ }^{1,2}$ \\ ${ }^{1}$ Universidade Federal de Mato Grosso do Sul, Campo Grande, MS, Brazil \\ ${ }^{2}$ Universidade Católica Dom Bosco, Campo Grande, MS, Brazil \\ ${ }^{3}$ Universidade Estadual de Mato Grosso do Sul, Aquidauana, MS, Brazil \\ ${ }^{4}$ Instituto Federal de Mato Grosso do Sul, Campo Grande, MS, Brazil \\ ${ }^{5}$ Instituto Federal de Mato Grosso do Sul, Aquidauana, MS, Brazil \\ ${ }^{6}$ Instituto Federal de Mato Grosso do Sul, Ponta Porã, MS, Brazil \\ ${ }^{7}$ University of Nebraska - Lincoln, EUA
}

\begin{abstract}
The objective of this paper is to investigate which combination of parameters for the fingerling counting software results in the smallest Mean Absolute Error (MAE) and smallest Root Mean Squared Error (RMSE). For this, an image dataset called FISHCV155V was created and separated into training and test sets, where different combinations of parameters for the software were tested. From the obtained results were extracted individual performance metrics for each combination of parameters, such as MAE, Mean Square Error (MSE) and RMSE. Video frames were analysed comparing the parameter combination that obtained the best and worst results, in order to investigate the influence of such parameters in the performance of the software. From such results, it was concluded that the best combination reached 5.99 MAE and 9.96 RMSE.

Index Terms - image/video analysis, automated counting, parameterization, computer vision.
\end{abstract}

\section{INTRODUCTION}

The production of fish, the second most produced protein in the world, is on the rise in Brazil. The forecast is for a growth of 100 percent in the production of fishing and aquaculture until the year 2025. The population growth and the increasing search for healthy food demand the adoption of technologies and advances in fish production [1], [2].

Brazil has $8,501 \mathrm{~km}$ of coastline, about $12 \%$ of the world's total fresh water and more than 2000 catalogued fresh water fish species, according to Buckup et. al [3]. This number reflects a large number of species with potential for culture. In this sense, natural or induced reproduction of fingerlings allows the sustainability of the pisciculture activity, since the exploitation of extractive fishing has generated an unbalance in the fish populations, even resulting in the risk of extinction

Corresponding author: Adair da Silva Oliveira Junior (email: adair.oliveira@ufms.edu.br). of some species. Additionally, the climatic conditions favor the production of species such as tilapia, which stands out for being the most consumed species in the world. Investments in innovation and research in recent years are factors that contribute to the predicted growth [4], [5].

Although pisciculture presents itself as an expanding farming activity all over Brazil and the world [6], many of the needed processes demand automatizing technologies to turn such activity more efficient, such as the Fingerling Counting System. Controlling fingerling stock regulation is a fundamental step in pisciculture, however having humans visually count each fingerling is a costly and tiring activity, creating the possibility of errors to appear.

Equipment with embedded computer vision-based software tools have been studied and developed in order to support this counting process $[7]-[11]$. Among this equipment and software reported, we highlight the fingerling counter developed by Inovisão group, whose main requirement refers to the counting of fingerlings of the species Pintado Real (Pseudoplatystoma corruscans) [12], [13].

We also highlight the importance of defining and adjusting parameters that have significant influence on the performance of software, especially counting software, as well as that reported for fingerling counting. However, most of the time, these parameters are set in an ad-hoc manner. In this sense, optimization of the parameters provides optimized values for variables implemented in the software in order to obtain the best result in the counts.

In this context, the aim of this paper is to investigate, within a bounded space of possibilities, which combination of parameters of the fingerling counting software results in the smallest mean absolute error and the smallest root mean 
squared error. The main contribution of this paper is the presentation of a methodology for evaluating the performance of the fingerling counting software for different combinations of parameters.

This paper is organized into five more sections. Section 2 presents related work. Section 3 presents a contextualization of the fingerling counter. Section 4 presents the methodologies for building the image dataset and executing the experiment. Section 5 presents and discusses the results obtained and, finally, Section 6 presents the conclusion.

\section{RELATED WORK}

Fish counters based on computer vision compete with three other types of counters: sound-based, resistive-based, and optic-based [7]. These counters are employed in all life stages of fish, meaning from its embryo stage up until it's adult life.

Fan and Liu [7] utilized background-subtraction techniques and skeletization together with LS-SVM (Least Squares Support Vector Machine) and BPNN (Back Propagation Neural Network) to count fish per video. The fish are placed in a vessel with water overlooked by a camera which will realize the image capturing. Seven geometric characteristics were used: area, perimeter, convex area, width, and height of the vessel, number, and size of the skeletons. An accuracy rate of 98.73\% was achieved, with the LS-SVM being superior to the BPNN.

Costa et al. [9] proposed a different system, in which two submerged cameras were present. Both of these cameras realized synchronous image capturing at the moment the fish of the Thunnus thynnus species were transported from a net to floating cages. Two frames were captured per second, which were processed by a software-based on artificial neural networks. These informations are utilized both to realize the counting and predict the length of fish.

Duan et al. [8] developed a computer vision-based method to realize the counting of eggs in dead fish. This was based on the principle that dead eggs float. It is highlighted that the advantage in the substitution of traditional methods of counting for a computer vision-based method is diminished handling of the eggs, meaning that it's a less invasive and less exhaustive method.

Klapp et al. [11] proposed a counting system for ornamental fish based on a hybrid technique that utilizes optic sensors and signal processing. It is highlighted that with this technique, the noise exerted by the water oscillation was diminished.

Hernández-Ontiveros [10] developed a fish counter utilizing an embedded system to process the images, which, in controlled lighting and species conditions, obtained $96 \%$ accuracy it's counting, with different species and sizes.

Garcia et al. [13] have already published preliminary results on the evaluation of the fingerling counter through its exploration of parameters. The best performance was obtained with an MSE of 2.65 and $\mathrm{R}$ squared 0.9803 . In contrast to that, we proposed an evaluation of a different dataset in this paper, and despite the same webcam, the recordings were with the smallest resolutions.

\section{Materials And Methods}

\section{A. Fingerling Counter}

The equipment responsible for the fingerling counting, was developed in [13], and basically consists of 4 main components: the structure, the lighting, the camera and the software. The structure is assembled in a way that it is inclined, around 11 to 13 degrees, through which the fingerlings slide with the help of water, which flows continuously. The inclination and water flow influence the velocity in which the fingerlings slide through the frame. If the velocity is too high, the captured images might not be sufficient to realize the fingerling tracking, while if the velocity is too low, the equipment will have a small fingerlings per hour count.

The lighting is also a important factor and there must be low reflection, as well as correcting any exterior changes, so that the resulting images are uniform, optimizing the focus and the contrast. The camera is coupled to the structure and captures the images. The parameters frame rate captured per second and resolution also directly influence the counting system, since if the rate is small, there will not be enough frames for tracking the fingerlings, on the other hand, if the rate is high, it will result in a greater consumption of computer resources, and may even make it impossible to count in real time. Figure 1 presents the structure of the fingerling counter.

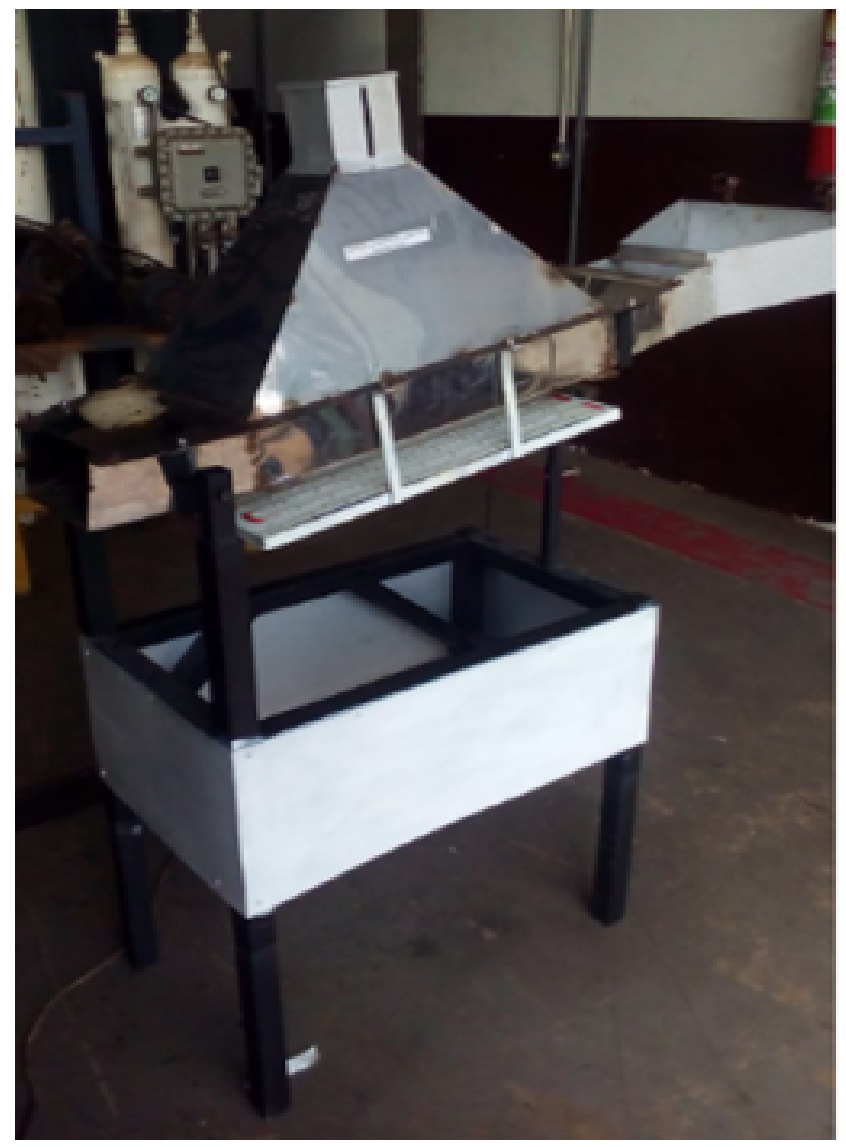

Fig. 1: Prototype of the fingerling counter structure. 
The fingerling counting software relies on the techniques of background subtraction segmentation, detection of connected components (Blobs), detection of contours and predictive filter of Kalman. The counting algorithm basically works on background subtraction segmentation and the detection of regions of interest and contours, which are tracked through the predictions made by the Kalman filter. The filter has parameters that define the counting pattern, such as the minimum area and the thresholding value for the detection of the connected components, in this case the fingerlings, and standard deviation of their area, for correction when there are agglomerations. In general terms, the algorithm performs the count through the following steps:

1) Segmentation by background subtraction;

2) Detection of regions of interest and contours, to label them as fingerling candidates;

3) Kalman Filter application on the candidates and association of detected regions of interest and contours in the new frame;

4) Checking the number of times a candidate was detected. If it reaches the value of a parameter, called candidate threshold, that candidate's label will be changed to fingerling;

5) Verification of the fingerling's position in relation to the count region. If it has crossed it, the calculation is performed.

The software has the following counting parameters:

- Distance between blobs: minimum distance between blobs of a frame. If a blob is found within this distance, both are associated as a single blob.

- Blob area: minimum area of a blob. Blobs with smaller areas are discarded.

- Blob Threshold: value for segmentation by thresholding of the blob.

- Candidate Threshold: how many times a candidate must be tracked in order to become a fingerling.

- Standard deviation: in case of agglomeration, an analysis of the blob area is done in order to determine if there is more than one fingerling.

- Minimum area: minimum area necessary for a blob to be counted as a fingerling. Similar to blob area, however smaller blobs can be identified for tracking reasons, with a verification being done during counting.

\section{B. Image Dataset FISHCV155V creation}

The creation of the image dataset was performed in the fish farming laboratory of Inovisão, which was set up in a partnership between Universidade Católica Dom Bosco-UCDB, Instituto Federal de Mato Grosso do Sul-IFMS and the company Projeto Pacu, through the fingerling counting equipment, described in previous subsection. Called FISHCV155V, the image dataset has 155 videos, captured by a Logitech HD Pro WebCam C920, with a resolution of $640 \times 320$ pixels at a rate of 30 frames per second. The number of fingerlings per video varied from 1 to 36 units. The recordings were conducted over
3 days between the months of July and September 2018. In addition to the number of fingerlings per video, it has the total mass of the fingerlings and the inclination of the equipment, a parameter that influences the speed at which the fingerlings pass through it. The methodology adopted in the capture of videos followed the following steps:

1) Separation of the fingerlings in 2 unused and already used containers;

2) Separation of a fingerling from container (1);

3) Capture images of fingerling in the equipment, associating the mass of the fingerling to the video;

4) Deposition of a fingerling in container (2);

5) If there is more than 1 fingerling in the container (2), capture the images of the fingerlings in the equipment, associating the aggregate mass to the video;

6) Repeat from item 2 to item 5 until there are no more fingerlings in the (1) container.

Due to the availability of fingerlings on the days in which the recordings were made, and the methodology adopted in capturing them, the numbers of videos of each quantity of fingerlings are different. The Figure 2 presents a histogram with the frequencies of the quantities of fingerlings per video.

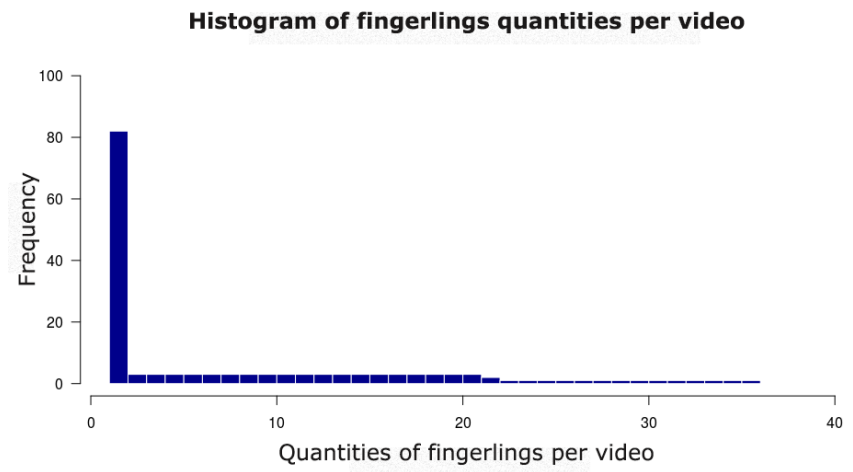

Fig. 2: Histogram of the numbers of fingerlings per video.

\section{Experimental Design}

The experiment was done on the FISHCV155V image dataset, divided into two sets, train, and test, with $70 \%$ and $30 \%$ being the percentage of separation, respectively. Multiple combinations of parameters for the counting software were tested to identify the best combinations. The parameters blob distance (bd), blob area (ba), blob threshold (bt), candidate threshold (ct), standard deviation (sd) and minimum area (ma) were combined, generating 324 different combinations, of whose values can be observed in Table I

From the obtained results, some performance metrics were extracted from each combination, them being: Mean Absolute Error (MAE), Mean Squared Errors (MSE), and Root Mean Squared Error (RMSE). The combinations that obtained the best results, i.e. achieved the lowest errors, were applied to the test set. In order to detect the possible influences of the parameters on the software, the combinations with the best and 
TABLE I: Values for the fingerling counting software used in the experiment.

\begin{tabular}{cc}
\hline Parameters & Values \\
\hline Distance between blobs & 62,64 and 66 \\
Blob area & 33,35 and 37 \\
Blob threshold & 68 and 70 \\
candidate threshold & 1 and 2 \\
Standard deviation & $0.7,0.9$ and 1.1 \\
Minimum area & $1.7,1.9$ and 2.1 \\
\hline
\end{tabular}

worst results during training were selected, to which videos were submitted and the behavior of the algorithm was analyzed frame by frame.

Figure 3 presents a flowchart with the general methodology applied to the fingerling counting experiment.

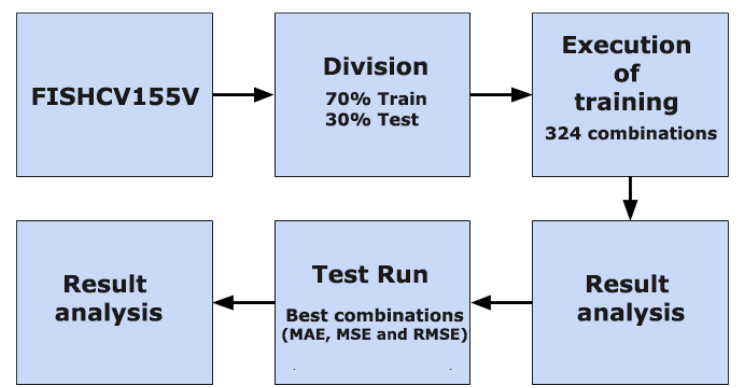

Fig. 3: Flowchart of the methodology applied in the fingerling counting experiment.

\section{RESUlTS AND Discussion}

Table III presents the results, containing the MAE, MSE, and RMSE metrics of the 20 best parameter combinations. It can be observed that the best result occurred when the blob area, blob threshold, the distance between blobs, candidate threshold, standard deviation, and minimum area parameters have the values $62,33,70,11.2$, and 1.7 respectively, with the mean absolute errors being 5.99, the mean squared errors 99.20, and the root mean squared errors 9.96.

Another fact that we can observe in Table $\amalg$ is that there are no changes in the metrics when the blob threshold and minimum area parameters alternate, generating than a draw between 9 combinations, causing other 8 combinations have the same performance as the appointed combination.

Table III presents the results, containing the MAE, MSE, and RMSE metrics of the 20 worst parameter combinations. We can observe that the worst result occurs when the blob area, blob threshold, the distance between blobs, candidate threshold, standard deviation, and minimum area parameters have the values $66,37,68,10.7$ and 2.1 respectively, with the mean absolute errors being 6.96, mean squared errors 119.81 and root mean squared errors 10.95 .

Table IV] presents the metrics, mean absolute errors, mean squared errors, and root mean squared errors, of the nine combinations which scored the best on the training set, for the test set.
TABLE II: Result of the 20 best parameter combinations for the training set.

\begin{tabular}{c|cccccc|ccc}
\hline \multirow{2}{*}{$\#$} & \multicolumn{7}{|c|}{ Parameters } & \multicolumn{3}{c}{ Metrics } \\
\cline { 2 - 9 } & ba & bt & bd & ct & ds & ma & MAE & MSE & RMSE \\
\hline 1 & 62 & 33 & 70 & 1 & 1,1 & 1,7 & 5,99 & 99,20 & 9,96 \\
2 & 62 & 33 & 70 & 1 & 1,1 & 1,9 & 5,99 & 99,20 & 9,96 \\
3 & 62 & 33 & 70 & 1 & 1,1 & 2,1 & 5,99 & 99,20 & 9,96 \\
4 & 62 & 35 & 70 & 1 & 1,1 & 1,7 & 5,99 & 99,20 & 9,96 \\
5 & 62 & 35 & 70 & 1 & 1,1 & 1,9 & 5,99 & 99,20 & 9,96 \\
6 & 62 & 35 & 70 & 1 & 1,1 & 2,1 & 5,99 & 99,20 & 9,96 \\
7 & 62 & 37 & 70 & 1 & 1,1 & 1,7 & 5,99 & 99,20 & 9,96 \\
8 & 62 & 37 & 70 & 1 & 1,1 & 1,9 & 5,99 & 99,20 & 9,96 \\
9 & 62 & 37 & 70 & 1 & 1,1 & 2,1 & 5,99 & 99,20 & 9,96 \\
10 & 64 & 33 & 70 & 1 & 1,1 & 1,7 & 6,01 & 100,03 & 10,00 \\
11 & 64 & 33 & 70 & 1 & 1,1 & 1,9 & 6,01 & 100,03 & 10,00 \\
12 & 64 & 33 & 70 & 1 & 1,1 & 2,1 & 6,01 & 100,03 & 10,00 \\
13 & 64 & 35 & 70 & 1 & 1,1 & 1,7 & 6,01 & 100,03 & 10,00 \\
14 & 64 & 35 & 70 & 1 & 1,1 & 1,9 & 6,01 & 100,03 & 10,00 \\
15 & 64 & 35 & 70 & 1 & 1,1 & 2,1 & 6,01 & 100,03 & 10,00 \\
16 & 64 & 37 & 70 & 1 & 1,1 & 1,7 & 6,01 & 100,03 & 10,00 \\
17 & 64 & 37 & 70 & 1 & 1,1 & 1,9 & 6,01 & 100,03 & 10,00 \\
18 & 64 & 37 & 70 & 1 & 1,1 & 2,1 & 6,01 & 100,03 & 10,00 \\
19 & 62 & 33 & 68 & 1 & 1,1 & 1,7 & 6,02 & 100,50 & 10,02 \\
20 & 62 & 33 & 68 & 1 & 1,1 & 1,9 & 6,02 & 100,50 & 10,02
\end{tabular}

TABLE III: Result of the 20 worst parameter combination for the training set.

\begin{tabular}{c|cccccc|ccc}
\hline \multirow{2}{*}{$\#$} & \multicolumn{7}{|c|}{ Parameters } & \multicolumn{3}{c}{ Metrics } \\
\cline { 2 - 9 } & ba & bt & bd & ct & ds & ma & MAE & MSE & RMSE \\
\hline 305 & 66 & 37 & 68 & 2 & 0,7 & 1,7 & 6,94 & 116,23 & 10,78 \\
306 & 66 & 37 & 68 & 2 & 0,7 & 1,9 & 6,94 & 116,23 & 10,78 \\
307 & 66 & 37 & 68 & 2 & 0,7 & 2,1 & 6,94 & 116,23 & 10,78 \\
308 & 62 & 33 & 68 & 1 & 0,7 & 1,9 & 6,95 & 119,60 & 10,94 \\
309 & 62 & 33 & 68 & 1 & 0,7 & 2,1 & 6,95 & 119,60 & 10,94 \\
310 & 62 & 35 & 68 & 1 & 0,7 & 1,7 & 6,95 & 119,60 & 10,94 \\
311 & 62 & 35 & 68 & 1 & 0,7 & 1,9 & 6,95 & 119,60 & 10,94 \\
312 & 62 & 35 & 68 & 1 & 0,7 & 2,1 & 6,95 & 119,60 & 10,94 \\
313 & 62 & 37 & 68 & 1 & 0,7 & 1,7 & 6,95 & 119,60 & 10,94 \\
314 & 62 & 37 & 68 & 1 & 0,7 & 1,9 & 6,95 & 119,60 & 10,94 \\
315 & 62 & 37 & 68 & 1 & 0,7 & 2,1 & 6,95 & 119,60 & 10,94 \\
316 & 66 & 33 & 68 & 1 & 0,7 & 1,7 & 6,96 & 119,81 & 10,95 \\
317 & 66 & 33 & 68 & 1 & 0,7 & 1,9 & 6,96 & 119,81 & 10,95 \\
318 & 66 & 33 & 68 & 1 & 0,7 & 2,1 & 6,96 & 119,81 & 10,95 \\
319 & 66 & 35 & 68 & 1 & 0,7 & 1,7 & 6,96 & 119,81 & 10,95 \\
320 & 66 & 35 & 68 & 1 & 0,7 & 1,9 & 6,96 & 119,81 & 10,95 \\
321 & 66 & 35 & 68 & 1 & 0,7 & 2,1 & 6,96 & 119,81 & 10,95 \\
322 & 66 & 37 & 68 & 1 & 0,7 & 1,7 & 6,96 & 119,81 & 10,95 \\
323 & 66 & 37 & 68 & 1 & 0,7 & 1,9 & 6,96 & 119,81 & 10,95 \\
324 & 66 & 37 & 68 & 1 & 0,7 & 2,1 & 6,96 & 119,81 & 10,95
\end{tabular}

Next, a frame-by-frame snippet analysis of 2 videos was performed for the parameters that obtained the best and worst results: position combination 1, noted in Table III, and position combination 324, noted in Table III in order to identify the possible errors in the counting algorithm. Figures containing the tracking of fingerlings are presented, in which the information regarding the count, represented by the value " $C$ ", and the frame, represented by the value "F", present in the upper right corner. The red traces indicate the predictions made by the Kalman filter, the unfilled circles with red borders represent the blobs. The circles with pink centers indicate fingerlings, and those with white centers indicate fingerling candidates.

In video 29 , between frames 80 and 81 , the algorithm was configured with the combination that resulted in the worst performance and realized the counting of 3 fingerlings, when it was actually supposed to count only one, as we can see in Figure 4b. Figure 4a shows the same frames of video 29, but with the algorithm set to the best match, where the algorithm performed the correct count. This is a false-positive problem. 
TABLE IV: Metrics of the best parameter combinations for the test set.

\begin{tabular}{cccccc|ccc}
\hline \multicolumn{7}{c|}{ Parameters } & \multicolumn{3}{c}{ Metrics } \\
\hline ba & bt & bd & ct & ds & ma & MAE & MSE & RMSE \\
\hline 62 & 33 & 70 & 1 & 1,1 & 1,7 & 5,00 & 83,00 & 9,11 \\
62 & 33 & 70 & 1 & 1,1 & 1,9 & 5,00 & 83,00 & 9,11 \\
62 & 33 & 70 & 1 & 1,1 & 2,1 & 5,00 & 83,00 & 9,11 \\
62 & 35 & 70 & 1 & 1,1 & 1,7 & 5,00 & 83,00 & 9,11 \\
62 & 35 & 70 & 1 & 1,1 & 1,9 & 5,00 & 83,00 & 9,11 \\
62 & 35 & 70 & 1 & 1,1 & 2,1 & 5,00 & 83,00 & 9,11 \\
62 & 37 & 70 & 1 & 1,1 & 1,7 & 5,00 & 83,00 & 9,11 \\
62 & 37 & 70 & 1 & 1,1 & 1,9 & 5,00 & 83,00 & 9,11 \\
62 & 37 & 70 & 1 & 1,1 & 2,1 & 5,00 & 83,00 & 9,11
\end{tabular}
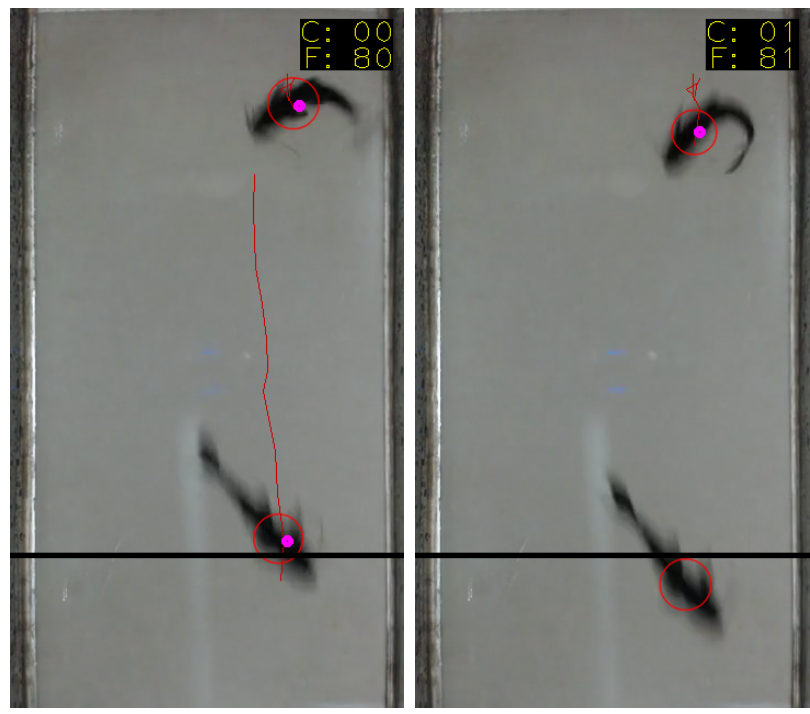

(a)
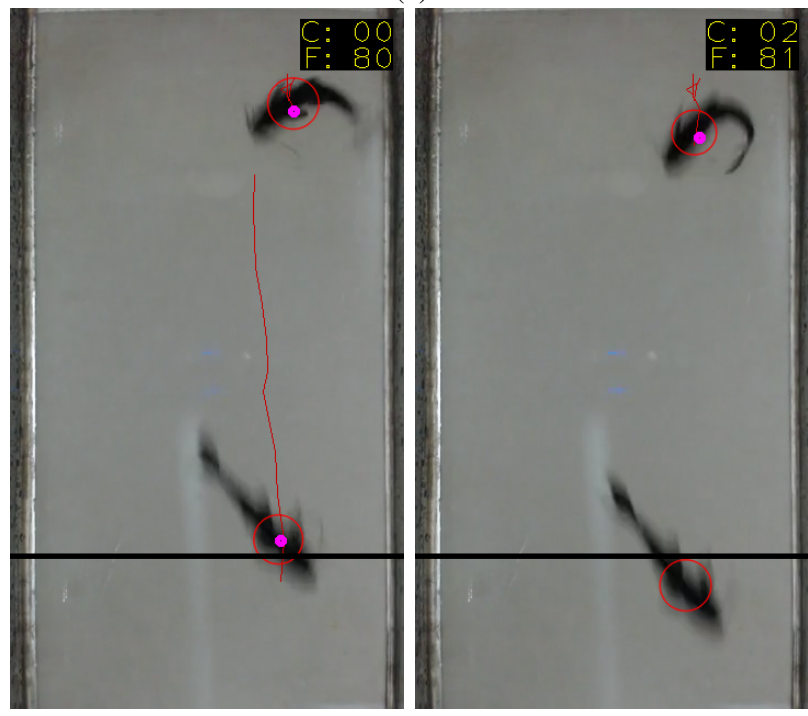

(b)

Fig. 4: Counting comparison on video 29, on frames 80 and 81 , between the parameter combinations that got the best and the worst result, where the worst combination performed a false-positive count.

As we can see in Tables $\coprod$ and $\amalg$ the combinations with the best and worst results have different values for the standard

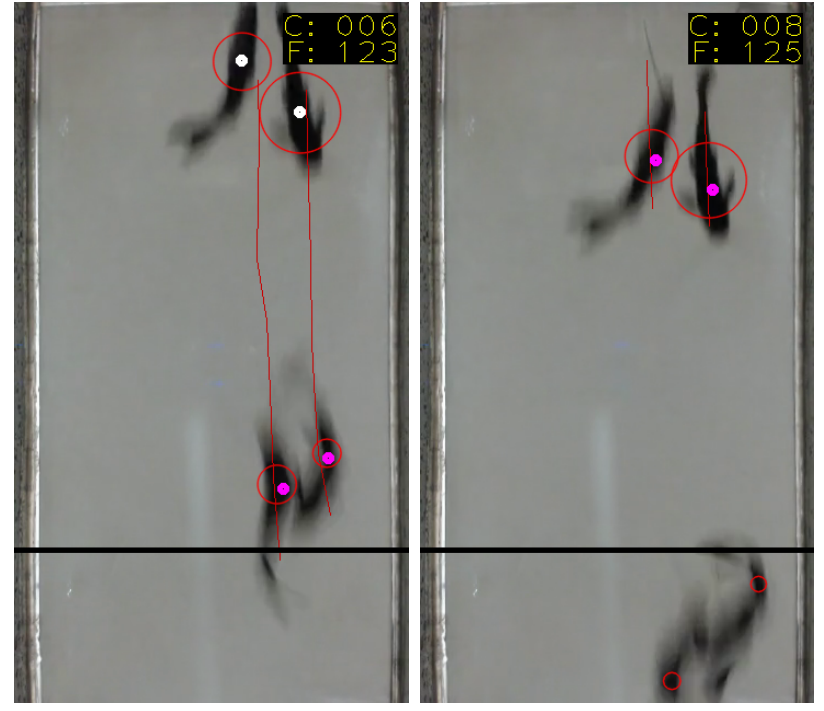

(a)

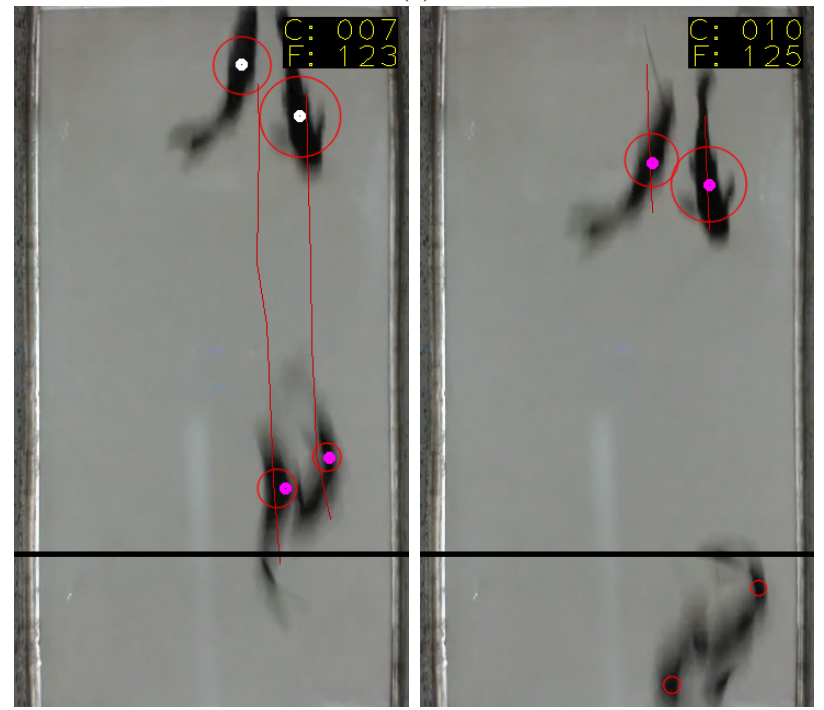

(b)

Fig. 5: Counting comparison on video 43, in frames 123 and 125 , between the parameter combinations that got the best and worst result, where the worst combination performed a falsepositive count. There is a difference in the count before frame 123 due to counting errors in the previous frames.

deviation parameter. This parameter defines the upper and lower limit that the blob area must have not to be considered too small to be a fish, an actual fish, or a cluster of fishes. Since the standard deviation is lower for the worst outcome combination compared to the best outcome combination, 0.7 and 1.1, respectively, the algorithm interprets the area of the blob as a cluster and performs the count of two fry instead of one.

In video 43, between frames 123 and 125, the algorithm, configured with the worst-case combination, counted three fry, when in fact it was supposed to count only two, as can be seen in Figure 5. This is a false-positive problem, and one of the 
possible causes is the relationship between the parameters of area of the blob, standard deviation and minimum area, which for these cases, when there is no agglomeration, these values are higher than ideal, causing the algorithm to count a larger number of fingerlings than are actually passing through the equipment.

\section{CONCLUSIONS}

From the resulting findings, it was concluded that the combination of parameters from the fingerling counting software, blob area, blob threshold, distance between blobs, candidate threshold, standard deviation and minimum area, with the values $62,33,70,1,1.1$ and 1.7 , respectively, has brought the smallest MAE, 5.99, and the smallest RMSE, 9.96, making such combination the best out of all 324 tested software parameter combinations, for the FISHCV155V dataset. For future work we intend to improve the identification and segmentation of the fingerling by means of semantic segmentation as well as to test another tracking mechanism by means of a particle filter in order to avoid clustering.

\section{ACKNOWLEDGMENT}

This paper has received support from the Universidade Católica Dom Bosco (UCDB), the Coordenação de Aperfeiçoamento de Pessoal de Nível Superior - Brasil (CAPES) - Finance Code 001 and Conselho Nacional de Desenvolvimento Científico e Tecnológico ( $\mathrm{CNPq}$ ) through research grants (p. 314902/2018-0), and from the Fundação de Apoio ao Desenvolvimento do Ensino, Ciência e Tecnologia do Estado de Mato Grosso do Sul (Fundect), granted through the concession term 131/2016, and by the partner company Projeto Pacu.

\section{REFERENCES}

[1] X. Zhou, "An overview of recently published global aquaculture statistics," FAO Aquaculture Newsletter, vol. 1, no. 56, p. 6, 2017.

[2] M. F. Brabo, L. F. S. Pereira, J. V. M. Santana, D. A. V. Campelo, and G. C. Veras, "Cenário atual da produção de pescado no mundo, no brasil e no estado do pará: ênfase na aquicultura, acta of fisheries and aquatic resources," Acta of Fisheries and Aquatic Resources, vol. 4, no. 2, pp. 50-58, 2016. [Online]. Available: https://www.tib.eu/de/ suchen/id/awi\%3Adoi\%7E10.2312\%252FActaFish.2016.4.2.50-58

[3] P. A. Buckup, N. A. Menezes, and M. S. Ghazzi, Catálogo das espécies de peixes de água doce do Brasil. Museu Nacional Rio de Janeiro, 2007, vol. 1.

[4] FAO, "Novo relatório da fao aponta que produção da pesca e aquicultura no brasil deve crescer mais de $100 \%$ até 2025," 2016. [Online]. Available: http://www.fao.org/brasil/noticias/detail-events/pt/c/423722/

[5] L. Sidonio, I. Cavalcanti, L. Capanema, R. Morch, G. Magalhães, J. Lima, V. Burns, A. J. Alves Júnior, and R. Mungioli, "Panorama da aquicultura no brasil: desafios e oportunidades," BNDES setorial, vol. 35, pp. 421-463, 2012.

[6] FAO, "PMA e OMS (2017) o estado da segurança alimentar e nutricional no mundo 2017: Construindo resiliência para a paz e segurança alimentar," $F A O$

[7] L. Fan and Y. Liu, "Automate fry counting using computer vision and multi-class least squares support vector machine," Aquaculture, vol. 380-383, pp. 91-98, 2013. [Online]. Available: https://www.sciencedirect.com/science/article/pii/S0044848612006175

[8] Y. Duan, L. H. Stien, A. Thorsen, Ø. Karlsen, N. Sandlund, D. Li, Z. Fu, and S. Meier, "An automatic counting system for transparent pelagic fish eggs based on computer vision," Aquacultural engineering, vol. 67, pp. $8-13,2015$.
[9] C. Costa, M. Scardi, V. Vitalini, and S. Cataudella, "A dual camera system for counting and sizing northern bluefin tuna (thunnus thynnus; linnaeus, 1758) stock, during transfer to aquaculture cages, with a semi automatic artificial neural network tool," Aquaculture, vol. 291, no. 3-4, pp. 161-167, 2009.

[10] J. Hernández-Ontiveros, E. Inzunza-González, E. García-Guerrero, O. López-Bonilla, S. Infante-Prieto, J. R. Cárdenas-Valdez, and E. TleloCuautle, "Development and implementation of a fish counter by using an embedded system," Computers and Electronics in Agriculture, vol. 145, pp. 53-62, 2018.

[11] I. Klapp, O. Arad, L. Rosenfeld, A. Barki, B. Shaked, and B. Zion, "Ornamental fish counting by non-imaging optical system for real-time applications," Computers and Electronics in Agriculture, vol. 153, pp. 126-133, 2018.

[12] P. Albuquerque, V. Garcia, A. Oliveira, T. Lewandowski, C. Detweiler, A. Barbosa Goncalves, C. Costa, M. Naka, and H. Pistori, "Automatic live fingerlings counting using computer vision," Computers and Electronics in Agriculture, vol. 167, p. 105015, 122019.

[13] V. Garcia, D. A. Sant'Ana, V. A. Garcia Zanoni, M. C. Brito Pache, M. H. Naka, P. L. França Albuquerque, T. Lewandowski, A. D. Silva Oliveira Junior, J. V. Araújo Rozales, M. W. Ferreira, E. Q. A. de Queiroz, J. C. Marino Almanza, and H. Pistori, "A new image dataset for the evaluation of automatic fingerlings counting," Aquacultural Engineering, vol. 89, p. 102064, 2020. [Online]. Available: https://www.sciencedirect.com/science/article/pii/S0144860919301700 